EPJ Web of Conferences 20, 01006 (2012)

DOI: $10.1051 /$ epjconf/20122001006

(C) Owned by the authors, published by EDP Sciences, 2012

\title{
Role of Anomalous Quark-Gluon Chromomagnetic Interaction in Hadron Physics
}

\author{
Nikolai Kochelev ${ }^{\mathrm{a}}$ \\ Bogoliubov Laboratory of Theoretical Physics, Joint Institute for Nuclear Research, Dubna, Moscow \\ region, 141980 Russia
}

\begin{abstract}
We calculate the contribution arising from nonperturbative quark-gluon chromomagnetic interaction to the high energy total quark-quark cross section. The estimation obtained within the instanton model of QCD vacuum leads to the conclusion that this type of interaction gives the dominating contribution to the Pomeron coupling with the light quarks and to gluon distribution in light hadrons at small virtualities of quarks and gluons.
\end{abstract}

\section{Introduction}

The gluon distribution in nucleon is one of the central quantities in particle physics which determines the high energy cross section values of the huge amount of important processes. In spite of the tremendous achievements in the last years in the measurement of this distribution, full understanding of the dynamics of gluons inside hadrons is absent so far (see reviews [1,2]). In the Regge theory the behaviour of the gluon distribution function at small Bjorken $x$ is controlled by the contribution coming from the Pomeron exchange which may have so-called "soft" and "hard" parts [3]. Usually, the hard Pomeron is associated with the perturbative BFKL regime [4] and the soft part is assumed to be originated from nonperturbative QCD dynamics [5]. Nonperturbative effects arise from the complex structure of QCD vacuum. The instantons are one of the well studied topological fluctuations of vacuum gluon fields which might be responsible for many nonperturbative phenomena observed in particle physics (see reviews [6,7]). Their possible importance in the structure of the Pomeron and gluon distribution was considered in quite different approaches [8], [9], [10], [11],[7] for the different approximations to the complicated quark-gluon dynamics in instanton vacuum. In particular, it was shown [12] that instantons lead to the appearance of anomalous chromomagnetic quark-gluon interaction (ACQGI). It was demonstrated that this new type of quark-gluon interaction might be responsible for the observed large single-spin asymmetries in various high energy reactions $[12,13]$. Furthermore, it gives a large contribution to the high energy quark-quark scattering cross section [14]. The first estimation of the effect of ACQGI on nucleon gluon distribution was made in [8] and small $\mathrm{x}$ behavior $g(x) \propto 1 / x$ corresponding to soft Pomeron was found. It was clear from that study that anomalous chromomagnetic interaction should also play an important role in the structure of Pomeron. Indeed, recently the model for soft Pomeron based on this interaction has been suggested [7].

In this paper, we consider the detailed structure of the Pomeron distribution with the special attention to the interplay between its perturbative and nonperturbative components.

\footnotetext{
${ }^{a}$ e-mail: kochelev@theor.jinr.ru
} 


\section{Anomalous chromomagnetic quark-gluon interaction}

In the general case, the interaction vertex of massive quark with gluon can be written in the following form:

$$
V_{\mu}\left(k_{1}^{2}, k_{2}^{2}, q^{2}\right) t^{a}=-g_{s} t^{a}\left[\gamma_{\mu} F_{1}\left(k_{1}^{2}, k_{2}^{2}, q^{2}\right)-\frac{\sigma_{\mu \nu} q_{v}}{2 M_{q}} F_{2}\left(k_{1}^{2}, k_{2}^{2}, q^{2}\right)\right],
$$

where the form factors $F_{1,2}$ describe nonlocality of the interaction, $k_{1,2}$ is the momentum of incoming

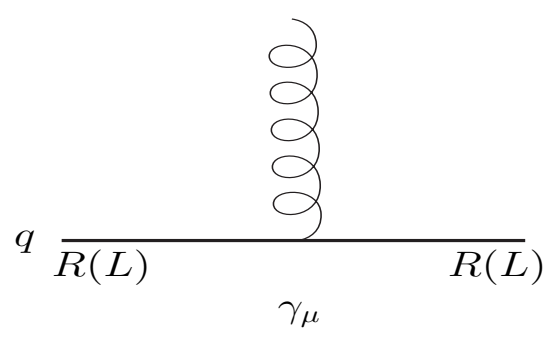

(a)

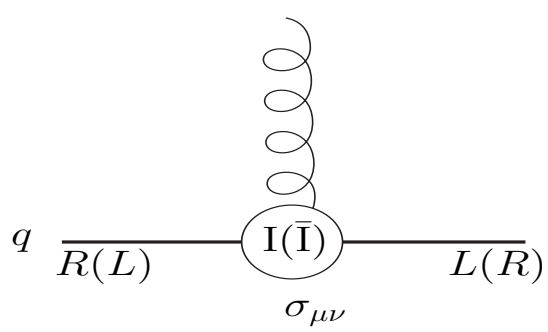

(b)

Fig. 1. The quark-gluon coupling: a) perturbative and b) nonperturbative. Symbols $R$ and $L$ denote quark chirality and symbol $I(\bar{I})$ denotes instanton (antiinstanton).

and outgoing quarks, respectively, and $q=k_{1}-k_{2}, M_{q}$ is the quark mass, and $\sigma_{\mu \nu}=\left(\gamma_{\mu} \gamma_{\nu}-\gamma_{\nu} \gamma_{\mu}\right) / 2$. In various applications to high energy reactions based on perturbative QCD (pQCD) it is usually assumed that only non-spin flip first term in Eq.(1) (Fig.1a) contributes and one can neglect the second term in this equation,Fig. 1b, because in the limit of the massless quark this term should be absent due to quark chirality conservation in massless pQCD. However, it has recently been shown that such assumption has no justification in nonperturbative QCD and second term might give in many cases even a dominant contribution to high energy reactions in comparison with the first one [12,14].

The cornerstone of this phenomenon is the spontaneous chiral symmetry breaking (SCSB) due to the complex topological structure of the QCD vacuum. Indeed, the instanton liquid model for QCD vacuum $[6,7]$ provides the mechanism for such breaking. That mechanism is related to the existence of quark zero modes in the instanton field. As the result of SCSB, the light quarks in nonperturbative QCD vacuum have the dynamical mass, $M_{q}$. Additionally, t'Hooft quark-quark interaction induced by quark-zero modes leads to the violation of $U(1)_{A}$ symmetry in strong interaction.

In high energy reactions one might naively expect the smallness of SCSB effects because of the energy $\sqrt{s} \gg M_{q}$. Indeed, it might be correct for the reactions where the dominating contribution comes from quark-exchange diagrams. Within the instanton model this type of diagrams is originated from the t'Hooft quark-quark interaction contribution. However, instantons also lead to specific quarkgluon chromomagnetic interaction [12] which is presented by the second term in Eq.(1) (Fig.1b). It is evident that this term should lead to a nonvanishing contribution to high energy reactions because it induces t-channel nonperturbative gluon exchange. The size of the contribution is determined by the value of anomalous quark chromomagnetic moment (AQCM) ${ }^{1}$

$$
\mu_{a}=F_{2}(0,0,0)
$$

We should point out that within the instanton model the shape of form factor $F_{2}\left(k_{1}^{2}, k_{2}^{2}, q^{2}\right)$ is fixed:

$$
F_{2}\left(k_{1}^{2}, k_{2}^{2}, q^{2}\right)=\mu_{a} \Phi_{q}\left(\left|k_{1}\right| \rho / 2\right) \Phi_{q}\left(\left|k_{2}\right| \rho / 2\right) F_{g}(|q| \rho),
$$

\footnotetext{
1 The definition of anomalous AQCM used in Eq.(2) differs by a factor of two from the corresponding quantity presented in [12] and [7].
} 
where

$$
\begin{aligned}
& \Phi_{q}(z)=-z \frac{d}{d z}\left(I_{0}(z) K_{0}(z)-I_{1}(z) K_{1}(z)\right), \\
& F_{g}(z)=\frac{4}{z^{2}}-2 K_{2}(z)
\end{aligned}
$$

are the Fourier-transformed quark zero-mode and instanton fields, respectively, and $I_{v}(z), K_{v}(z)$, are the modified Bessel functions and $\rho$ is the instanton size.

The value of AQCM is determined by the effective density of the instantons $n(\rho)$ in nonperturbative QCD vacuum [12]:

$$
\mu_{a}=-\pi^{3} \int \frac{d \rho n(\rho) \rho^{4}}{\alpha_{s}(\rho)} .
$$

The shape of instanton density in the form

$$
n(\rho)=n_{c} \delta\left(\rho-\rho_{c}\right)
$$

leads to AQCM which is proportional to the packing fraction of instantons $f=\pi^{2} n_{c} \rho_{c}^{4}$ in vacuum

$$
\mu_{a}=-\frac{\pi f}{\alpha_{s}\left(\rho_{c}\right)}
$$

By using the following relation between parameters of the instanton model [15]:

$$
f=\frac{3}{4}\left(M_{q} \rho_{c}\right)^{2},
$$

we obtain

$$
\mu_{a}=-\frac{3 \pi\left(M_{q} \rho_{c}\right)^{2}}{4 \alpha_{s}\left(\rho_{c}\right)} .
$$

This formula coincides with the result for AQCM presented in Eq.(7.2) in the paper by Diakonov [7] and shows the direct connection between AQCM and SCSB phenomena. The dimensionless parameter $\delta=\left(M_{q} \rho_{c}\right)^{2}$ is one of the main parameters of the instanton model. It is proportional to the packing fraction of instantons in QCD vacuum $\delta \propto f \ll 1$, Eq.(8), and is rather small. For a fixed value of average instanton size $\rho_{c}^{-1}=0.6 \mathrm{GeV}$ it changes from $\delta^{M F}=0.08$ for $M_{q}=170 \mathrm{MeV}$ in the mean field approximation [6] to $\delta^{D P}=0.33$ for $M_{q}=345 \mathrm{MeV}$ within Diakonov-Petrov model (DP) [16]. For the strong coupling constant at the scale of instanton average size [6],[7]

$$
\alpha_{s}\left(\rho_{c}\right) \approx 0.5
$$

we obtain the following values for AQCM:

$$
\mu_{a}^{M F} \approx-0.4, \quad \mu_{a}^{D P} \approx-1.6
$$

in the mean field approximation and in the DP approach, respectively. We would like to emphasize that in spite of the strong dependence of AQCM on the value of the effective quark mass in QCD vacuum, AQCM is very large in the wide interval the possible changing of instanton model parameters. The origin of this peculiarity is in the large numerical factor in front of $\delta$ in Eq.(9) for AQCM. Indeed, this formula can be rewritten in the following form:

$$
\mu_{a}=-\frac{3}{8} S_{0} \delta
$$

where $S_{0}=2 \pi / \alpha_{s}(\rho)$ is the Euclidean instanton action. The typical value of this action is very large $[6,7]$

$$
S_{0} \approx 10 \div 15
$$




\section{EPJ Web of Conferences}

and leads to the compensation of the $\delta$ smallness effect on AQCM.

Within the instanton model approach the first term in Eq.(1) is related to the nonzero mode contribution to quark propagator in the instanton field. The nonzero modes contribution to quark propagator can be approximated with high accuracy by perturbative propagator [6]. Due to zero mode dominance for the light quarks, [6], we can expect that for the light quarks this sort of contribution should be suppressed in comparison with the second term in Eq.(1). However, for heavy quark the first term should dominate because there are no zero modes for heavy quark in the instanton field. Furthermore, instanton induced form factors in the chromomagnetic part of interaction suppress the contribution of the second term for highly virtual quark and/or gluon. Therefore, form factor in the first term in Eq.(1) might be chosen in the form

$$
F_{1}\left(k_{1}^{2}, k_{2}^{2}, q^{2}\right)=\Theta\left(\left|k_{1}^{2}\right|+\left|k_{2}^{2}\right|+\left|q^{2}\right|-3 \mu^{2}\right),
$$

where $\mu$ is the factorization scale between perturbative and nonperturbative regimes. In our estimation below we will use $\mu \approx 1 / \rho_{c} \approx 0.6 \mathrm{GeV}$.

\section{Pomeron structure}

Let us estimate the contribution of the vertex, Eq.(1), to the total high energy quark-quark scattering cross section. The leading diagrams contributing to the non-spin flip amplitude of $q-q$ scattering are shown in Fig. 2 and for colorless t-channel exchange presents the model of the Pomeron. The imaginary part of the total forward scattering amplitude gives the total quark-quark cross-section.

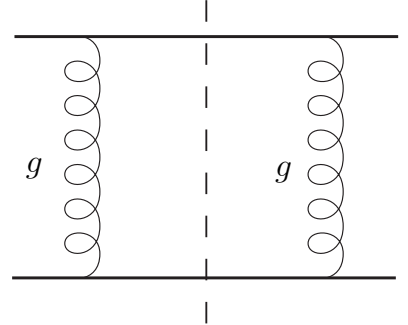

$(a)$

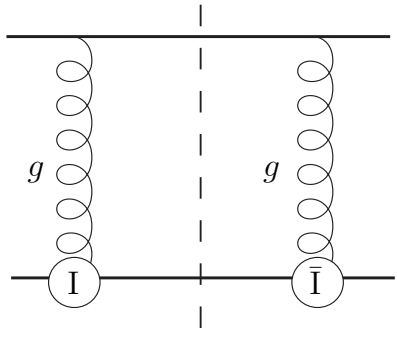

(b)

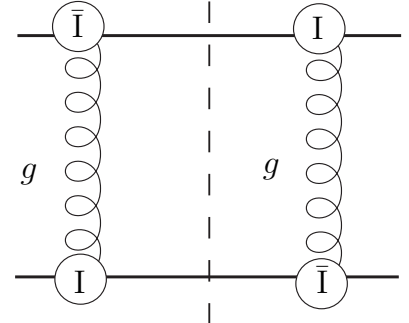

$(c)$

Fig. 2. The fine Pomeron structure in the model with perturbative interaction and nonperturbative ACQGI: a) perturbative contribution, b) interference perturbative and nonperturbative vertices, c) nonperturbative contribution. The symbol $I(\bar{I})$ denotes instanton (antiinstanton).

So, in our model Pomeron includes the pure perturbative exchange (Fig.2a), nonperturbative (Fig.2c) diagrams and the mixed graph (Fig.2b).

By using the relation, Eq.(9), the total contribution to quark-quark cross section for the quarks with small virtualities is

$$
\sigma^{\text {total }}=\sigma^{\text {pert }}+\sigma^{\text {mix }}+\sigma^{\text {nonpert }},
$$

where

$$
\begin{aligned}
\sigma^{i} & =\int_{q_{\min }^{2}}^{\infty} \frac{d \sigma^{i}(t)}{d t} d q^{2}, \\
\frac{d \sigma(t)^{p e r t}}{d t} & =\frac{8 \pi \alpha_{s}^{2}\left(q^{2}\right)}{9 q^{4}} \\
\frac{d \sigma(t)^{m i x}}{d t} & =\frac{\alpha_{s}\left(q^{2}\right) \pi^{2}\left|\mu_{a}\right| \rho_{c}^{2} F_{g}^{2}\left(|q| \rho_{c}\right)}{3 q^{2}}
\end{aligned}
$$




$$
\frac{d \sigma(t)^{\text {nonpert }}}{d t}=\frac{\pi^{3} \mu_{a}^{2} \rho_{c}^{4} F_{g}^{4}\left(|q| \rho_{c}\right)}{32}
$$

where $q^{2}=-t$ and $q_{\min }^{2} \approx 1 / \rho_{c}^{2}$ for perturbative and mixed contributions and $q_{\min }^{2}=0$ for pure nonperturbative (Fig.2c) contribution.

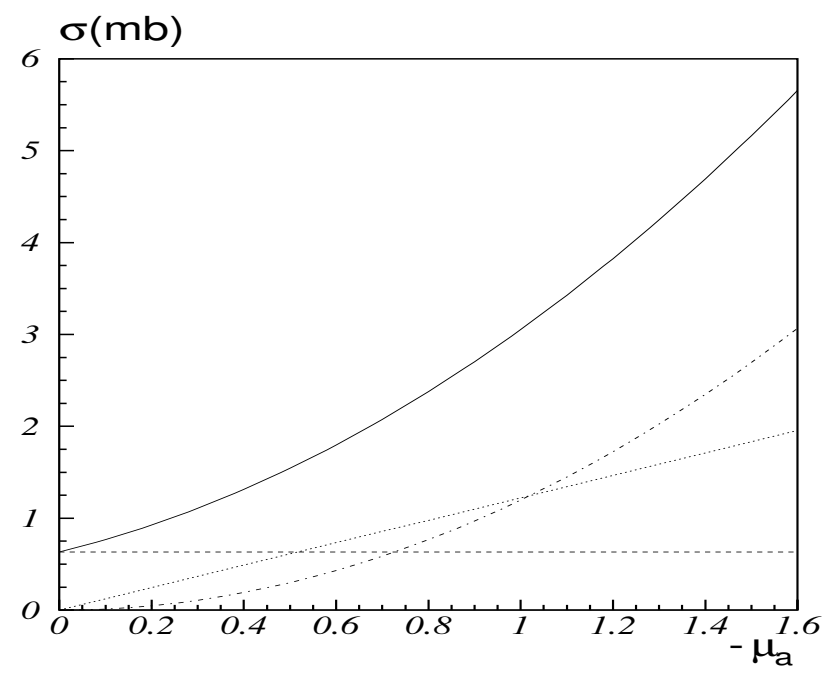

Fig. 3. The contibution to the total quark-quark cross section as a function of AQCM: perturbative (dashed line), mixed (dotted line), nonperturbative (dashed-dotted line) and their sum (solid line).

For the strong coupling constant, the following parametrization was used for the case $N_{f}=3$ :

$$
\alpha_{s}\left(q^{2}\right)=\frac{4 \pi}{9 \ln \left(\left(q^{2}+m_{g}^{2}\right) / \Lambda_{Q C D}^{2}\right)},
$$

where $\Lambda_{Q C D}=0.280 \mathrm{GeV}$ and the value $m_{g}=0.88 \mathrm{GeV}$ was fixed from the requirement $\alpha_{s}\left(q^{2}=\right.$ $\left.1 / \rho_{c}^{2}\right) \approx \pi / 6$ [7]. This form describes the frozen coupling constant in the infrared region, $\alpha_{s}\left(q^{2}\right) \rightarrow$ constant as $q^{2} \rightarrow 0$.

The result of calculation of the different contributions to the total quark-quark scattering cross section is presented in Fig. 3 as a function of AQCM. It is evident that within the interval $0.4<\left|\mu_{a}\right|<$ 1.6 the main contribution comes from the terms related to the anomalous quark-gluon chromomagnetic interaction. Recently, the effects of nonzero AQCM in hadron spectroscopy has been considered (see [17] and references therein). It was shown that the value of

$$
\mu_{a}=-1
$$

is favoring to describe the fine structure of hadron spectrum. This value of AQCM corresponds to dynamical quark mass $M_{q}=280 \mathrm{MeV}$. This mass is in agreement with recent result of analysis of dressed-quark propagator within DSE approach involving the lattice-QCD data from [18]. We will adopt this value in our estimations below. For that set of parameters the total quark-quark cross section $\sigma_{q q}^{\text {total }}=3.05 \mathrm{mb}$ is the sum of the following partial cross sections:

$$
\sigma_{q q}^{p e r t}=0.63 m b, \quad \sigma_{q q}^{m i x}=1.22 m b, \sigma_{q q}^{\text {nonpert }}=1.21 \mathrm{mb},
$$

and it is not far away from "experimental" quark constituent model value $\sigma_{q q}^{\text {exp }} \approx 4 \mathrm{mb}$, which is needed to describe the inelastic proton-proton and proton-antiproton cross-sections; $\sigma_{P P(\bar{P})}^{i n}=36 \mathrm{mb}$ in the 
energy range where they are approximately constant. One may expect also an additional contribution to the total cross section arises from the multigluon and multiquark emission induced by the quarkgluon-instanton vertex. It will bring our esimation to the experimental value. It follows from Eq.(20) that the contribution to the quark-quark cross section due to non-perturbative chromagnetic quarkgluon interaction is about $80 \%$ and the contribution from pure perturbative exchange is about $20 \%$ and quite small. Therefore, within our model the dynamics of soft Pomeron is determined not by the $\gamma_{\mu}$-like quark-gluon vertex (Fig.1a) as in most conventional models for the Pomeron, but by the $\sigma_{\mu \nu}$ vertex pictured in Fig.1b. The widely assuming statement is that the difference in the dynamics of soft and hard Pomerons comes from the difference in their dependence on such kinematic variables as total energy and transfer momenta. From our point of view, the main source of difference between two exchanges arises from a completely different spin structure of quark-gluon interaction inside the Pomeron exchange.

\section{Acknowledgments}

The author is very grateful to organizers of HNP2011 Conference for the warm hospitality extended to him. This work was supported in part by RFBR grant 10-02-00368-a, and by Belarus-JINR grant.

\section{References}

1. P. V. Landshoff, arXiv:0903.1523 [hep-ph].

2. I. P. Ivanov, N. N. Nikolaev and A. A. Savin, Phys. Part. Nucl. 37 (2006) 1 [arXiv:hep$\mathrm{ph} / 0501034]$.

3. P. V. Landshoff, arXiv:hep-ph/0209364.

4. E. A. Kuraev, L. N. Lipatov and V. S. Fadin, Sov. Phys. JETP 45 (1977) 199 [Zh. Eksp. Teor. Fiz. 72 (1977) 377]; I. I. Balitsky and L. N. Lipatov, Sov. J. Nucl. Phys. 28 (1978) 822 [Yad. Fiz. 28 (1978) 1597]; L. N. Lipatov, Sov. Phys. JETP 63, 904 (1986) [Zh. Eksp. Teor. Fiz. 90, 1536 (1986)].

5. P. V. Landshoff and O. Nachtmann, Z. Phys. C 35 (1987) 405.

6. T. Schäfer and E.V. Shuryak, Rev. Mod. Phys. 70 (1998) 1323.

7. D. Diakonov, Prog. Par. Nucl. Phys. 51 (2003) 173.

8. N. I. Kochelev, arXiv:hep-ph/9707418.

9. E. V. Shuryak and I. Zahed, Phys. Rev. D 62 (2000) 085014.

10. D. E. Kharzeev, Y. V. Kovchegov and E. Levin, Nucl. Phys. A 690 (2001) 621.

11. A. E. Dorokhov and I. O. Cherednikov, Annals Phys. 314 (2004) 321.

12. N. I. Kochelev, Phys. Lett. B426 (1998) 149.

13. I. O. Cherednikov, U. D’Alesio, N. I. Kochelev and F. Murgia, Phys. Lett. B 642 (2006) 39.

14. N. Kochelev, JETP Lett. 83 (2006) 527.

15. A. E. Dorokhov, N. I. Kochelev and S. V. Esaibegian, Phys. Atom. Nucl. 59 (1996) 2006 [Yad. Fiz. 59 (1996) 2081].

16. D. Diakonov and V. Y. Petrov, Nucl. Phys. B 272 (1986) 457.

17. D. Ebert, R. N. Faustov and V. O. Galkin, arXiv:0903.5183 [hep-ph].

18. M. S. Bhagwat and P. C. Tandy, AIP Conf. Proc. 842 (2006) 225 [arXiv:nucl-th/0601020]. 